\title{
Immunodiagnosis of tuberculosis: new questions, new tools conference 2008
}

\author{
Maria L Gennaro ${ }^{1 * \dagger}$, T Mark Doherty ${ }^{2+}$ \\ From Immunodiagnosis of Tuberculosis: New Questions, New Tools \\ Virginia, VA, USA. 21-23 September 2008
}

\begin{abstract}
Human infection with Mycobacterium tuberculosis exists as a spectrum of conditions ranging from asymptomatic infection to active disease. Novel, accurate tuberculosis immunodiagnostics have been introduced over the last decade, but it remains challenging to timely diagnose active disease and to accurately distinguish asymptomatic M. tuberculosis infection from immune memory resulting from a prior infection eradicated by the host response. The conference titled Immunodiagnosis of Tuberculosis: New Questions, New Tools, which was held on September 21-23, 2008 in Virginia Beach, Virginia, United States, brought together basic scientists and clinical experts to discuss recent progress in tuberculosis research and diagnosis. Global analyses of $M$. tuberculosis biology and the host immune response, with emphasis on systems approaches to the study of host-pathogen interactions, were presented. Moreover, conference participants discussed new tests in the pipeline and reviewed new technologies leading to novel assay formats. The discussion included technologies ranging from simple, inexpensive point-ofcare tests to automated molecular platforms for detection of multiple infections based on the "lab on a chip" concept. It was also recognized that the utility of any new diagnostic relies on laboratory capacity, accessibility, costs, and test deployment. The conference included lessons from the field. For example, the application of existing technologies to neglected areas, such as diagnosis in children and $\mathrm{HIV}^{+}$populations, was discussed.
\end{abstract}

\section{Introduction}

The first international meeting on new biomarkers and tools for the immunodiagnosis of $M$. tuberculosis infection and disease, entitled Immunodiagnosis of Tuberculosis: New Questions, New Tools, was held September 21 to 23, 2008 in Virginia Beach, Virginia, United States. The objective of the conference was to integrate the most recent knowledge generated through basic, translational, and clinical research that could lead to novel, improved immunodiagnostic tools for tuberculosis (TB). About 150 researchers from academia and the private sector attended the seminar from over 26 countries, including many developing nations. The agenda included 20 scientific presentations and a keynote

\footnotetext{
* Correspondence: gennarma@umdnj.edu

+ Contributed equally

${ }^{1}$ Public Health Research Institute, New Jersey Medical School, Newark, New Jersey, USA

Full list of author information is available at the end of the article
}

address by the director of the Stop TB Department at the World Health Organization (WHO).

TB is still a major killer: one person dies from this infectious disease every 15 seconds, somewhere in the world. The interaction between the human host and the causative agent of TB, the intracellular pathogen $\mathrm{Myco}$ bacterium tuberculosis, is characterized by the ability of the host immune response to control infection without causing sterilization and of the pathogen to withstand expression of host immunity by changing its metabolic and growth state. The result is an asymptomatic, chronic (latent) infection that may last for the lifetime of the host. In $5-10 \%$ of immunocompetent, infected individuals the host immune response loses control of infection at some time in the life of the individual: tubercle bacilli resume growth, and tuberculosis (TB) develops. Diseased individuals are usually infectious some time during disease. Their timely identification is critical to the success of chemotherapy and to curbing transmission of infection. Consequently, the 
development of disease in latently infected individuals is a major problem for TB control, as it means that new disease foci can arise outside of identified at-risk populations.

Until recently, century-old, often inaccurate methods have been the mainstay of diagnosis of active disease and latent infection [1]. Recent effort has resulted in new immunologic tests for latent infection, while diagnosis of active disease typically relies on detecting tubercle bacilli or their products in the patient's escreate (sputum). Neither old nor new tests, however, can predict progression from latent infection to active disease, a crucial stage for treatment and infection control. The need for better diagnostics for TB has been strongly advocated by The Global Plan to Stop TB 2006-2015 (http://www.stoptb.org/global/plan), which calls for universal access to high-quality diagnosis and research towards new diagnostics. Moreover, academia, industry, governmental agencies, and private agencies have expressed a renewed interest in TB diagnosis. However, achieving new, accurate TB diagnostics faces formidable challenges that are both scientific and operational. Overcoming them requires effective multi-disciplinary communication and collaboration within the scientific community.

The international conference "Immunodiagnosis of Tuberculosis: New Questions, New Tools" was planned as an authoritative venue for establishing partnerships and consortia leading to the next generation of TB immunodiagnostics. The speaker roster was of world-class quality, and the unique combination of themes concerning basic and translational research, field studies, and novel technologies was designed to achieve the conference goals. With its structure of plenary sessions and poster sessions, the meeting encouraged discussion and fostered collaboration among scientists in basic research, translational research, field studies, and industry to link research on biomarker discovery to a deeper understanding of the biology of the pathogen, the immune response of the host, and the host-pathogen interactions leading to the expression of immunological markers specific for various stages of infection (latent infection, progression, and disease). Moreover, the conference aimed at establishing links between basic and translational research (i) by discussing how new markers of infection and disease identified by basic research could be evaluated in field studies and (ii) by reviewing cutting-edge technologies that could lead to a new generation of immunodiagnostic tests. The goals of the conference were accomplished by integrating (i) discussion of the biological events associated with expression of immune markers of recent infection, latent chronic infection, progression, and active disease, (ii) the identification of translational research opportunities, (iii) the presentation of potential innovative new assay formats, and (iv) the definition of challenges at the preclinical and clinical stages of test development. Particular attention was given to pediatric $\mathrm{TB}$, which poses unique diagnostic challenges, since children typically present a different disease spectrum than adults and are likely express immune markers differently from adults due to the immaturity of the child's immune response.

\section{Summary of oral presentations during the plenary sessions}

Keynote address: Tuberculosis is a global health issue

Mario Raviglione, director of the World Health Organization's (WHO) Stop TB program, opened the conference with WHO's perspective on the global TB situation, discussing the status of the epidemic, progress that has been made toward eliminating this disease as a major public health problem worldwide by 2050, and the critical and urgent need to increase funding for research to achieve this goal. [2] Raviglione emphasized that a ten-year funding gap for global TB research and development has been a major impediment to scientific progress. A “TB Research Movement” has been conceived to accelerate progress, the goal of which is to stimulate investments, support efforts, accelerate implementation, and expand the existing portfolio across the research continuum to ensure development of tools that will be conducive to global elimination of TB by 2050. [2] Its objectives are 1) to provide leadership and advocacy to mobilize increased resources in support of a coherent and comprehensive global TB research agenda, and 2) to provide a forum for funders and implementers of TB research to coordinate plans and actions, with the result of ensuring that research needs are addressed, opportunities prioritized, and gaps filled. [2]

\section{Basic TB biology: current knowledge, bottlenecks and challenges}

The meeting began with a review of basic TB biology that focused on current bottlenecks and challenges in basic research. Douglas Young opened the session with a discussion of the biology of latent $\mathrm{TB}$, which he argued is essential to understanding the fundamental biology of TB. He specifically addressed current knowledge about why $M$. tuberculosis is able to resist the host's ability to eradicate it over a long period of time. [3] The commonly-used definition of latent TB-antigen-specific $\mathrm{T}$-cell response without clinical symptoms -is very broad and, Young argued, may in fact lead us away from the consideration that latent TB may not act the same way in every patient. [3] Young also discussed key scientific questions including whether everyone infected with latent TB harbors viable bacteria, and if 
so, where these bacteria are located in the body and what they are doing. [3]

Markus Wenk then discussed the cell biology of intracellular pathogens that has characterized the importance of lipids at various stages in host-pathogen interactions. [4] Lipids appear to be "gatekeepers" in important chemical reactions involving cell signaling during pathogen docking, invasion, and movement in and out of cells. Lipids may be viable targets for regulation of the hostpathogen interaction. [4]

Sebastien Gagnuex discussed evolutionary forces that have shaped the genetic diversity in $M$. tuberculosis, in particular natural selection, host migration, and changes in host demography. [5] JohnJoe McFadden then discussed the value of system approaches to uncover characteristics of the TB bacillus grown in vivo that could ultimately be useful as targets for new immunodiagnostic approaches. This approach, he argued, requires an in silico model of linked metabolic pathways to identify genes and phenotypes and predict their interactions. [6]

Lastly, Steven Elledge presented a functional genomics approach to viral-host interactions with HIV. New screening methodologies developed over the past five years, based on the novel understanding of the biology of double-stranded DNA and RNA interference, he argued, now provide scientists with the tools to carry out genetic screens of mammalian cells to identify host functions involved in the interaction with a particular pathogen. [7]

\section{Basic TB immunology: current knowledge, bottlenecks and challenges}

The second portion of the conference focused on the host side of TB, covering challenges in TB immunology. Stefan Kauffman presented research related to using the metabolic profile of the host response to $\mathrm{TB}$ as a basis for the rational design of vaccines and biomarkers. [8] Mark Doherty spoke about the potential for identifying biomarkers of infection, citing examples that indicated how they could be useful in separating latent and acute TB. [9] Padmini Salgame posed the question of how helminth infections, which are endemic in areas most affected by $\mathrm{TB}$, affect progression to active $\mathrm{TB}$ or reactivation of latent TB. She discussed her work with the Th1/Th2 paradigm to further examine this relationship. Maria Gennaro spoke about how high-throughput methods have facilitated the study of antibody responses on the genome-scale, and she discussed implications of this technology for monitoring disease progression using serology and profiling the biology of the tubercle bacilli in the human host. [10] Muireann Coen discussed the potential of translating metabolomics discoveries into tools for diagnosis and systems monitoring, and new technologies in biomarker discovery. [11]

\section{Pediatric tuberculosis}

Joseph Bellanti introduced the differences in the pediatric immune system that make children more likely to develop severe $\mathrm{TB}$, and he cited the need for obtaining new knowledge about the relationship between immune maturation and developmental immune deficiencies associated with infection. [12] Anneke Hesseling then spoke about pediatric TB from a clinical epidemiological perspective, based on her studies in a highly endemic setting in South Africa. She emphasized the need for improved diagnostics, improved programs to prevent TB and $\mathrm{TB} / \mathrm{HIV}$ co-infection, and shorter treatment regimens that target children. [13]

\section{Field studies/clinical trials: present and future}

Richard Menzies critically analyzed the methods used to evaluate the effectiveness of new diagnostic tests in the field, and he outlined a standardized approach that should be used to provide the necessary evidence of safety and efficacy. [14] Sandra Arend discussed the properties of a tuberculosis-specific skin test that showed promise in preliminary studies as a safe diagnostic that produced a readable skin test response and did not easily sensitize. [15] Peter Andersen discussed the value of IGRA-based diagnosis of infection and prediction of active disease, particularly in asymptomatically infected people. [16]

\section{New technologies: diagnostic assays for resource-rich and resource-limited settings}

Mark Perkins discussed the potential and limitations of seroimmunodiagnosis for case detection in the context of global disease care and control, including the need for either sensitivity or specificity in order for seroimmunodiagnostics to be useful globally. Philip Felgner discussed the use of microarray chip analysis to profile immunity to infectious diseases. His research demonstrates the usefulness of the technology to differentiate immune states during infection. His goal is to develop multi-variant microarray chips that contain several antigens. Mario Roederer discussed the application of many of his team's techniques for HIV/SIV vaccine development to TB, particularly with flow cytometry's ability to measure immune responses at the $T$ cell subset level. [17] Abraham Lee presented novel microfluidic technologies that could be of great value for use in TB serodiagnosis or as platforms for cell and biomolecular assays. [18] These technologies have properties that may make them globally useful. They include low costs, precision, speed, and integration capabilities to assess multiple parameters per sample. [18] Lastly, Adrian Ozinsky discussed the strengths and weaknesses of an application of microfluidic tools utilizing parallel single cell assays for TB diagnostics. While the application shows 
potential with its sensitivity, more research is needed to further define correlates of immunity and identify relevant cells for measurement.

\section{Research recommendations that emerged from the conference}

\section{Research priorities}

- Diagnostic tools that differentiate latent M. tuberculosis infection from acute infection

- Diagnostic tools that predict durable cure during therapy and risk of relapse after therapy

- Rapid, point of care identification of drug-resistant $T B$

- Prognostic tools to predict risk of reactivation or progress to disease

- Biomarkers predicting vaccine efficacy/correlates of protection

Areas for innovation and partnerships and cooperation

$\circ$ Leverage existing and planned clinical trials to test potential biomarkers of disease and protection

- Leverage new screening methodologies developed over the past five years for genetic screens of mammalian cells

- Learn from HIV vaccine development

- Leverage technology such as microfluidics to improve field diagnostic capabilities

- Establish systems biology partnerships with an emphasis on metabolomics to identify novel biomarkers

\section{Conclusions}

The recommendations above highlight issues that are critical for research required to provide new tools for TB diagnosis. The conference also acknowledged that "turning research into tools" requires acknowledging and correcting the limited success (or even outright failure) experienced with the deployment of technologies to the field. Recent advances in TB diagnosis have produced tools that were not designed with pricing or performance characteristics suitable for developing countries. In addition, we need to recognize that market forces have typically failed to deliver products that are primarily needed for poor patients in resource-limited countries; we need to develop pricing mechanisms or supply strategies to address this. Moreover, the experience of HIV and malaria, two global diseases for which cheap, simple, point-of-care tests are available, has revealed the many weaknesses of the developing countries' healthcare systems in management, financial and human resources, laboratory capacity, and quality assurance. Thus, public-private partnerships need to be supported to move forward technology in areas where private initiatives have not emerged (for example, simple point-of-care diagnostics) and existing diagnostic capacity needs to be identified and strengthened.

To carry out research, development, and deployment of new diagnostics, additional financial resources need to be identified. The Global Plan to Stop TB 2006-2015 estimated that at least 516 million USD are needed to strengthen TB diagnostics, yet TB diagnostics development received only a tenth of that, according to the 2009 Treatment Action Group and Stop TB Partnership reports [19]. While expanded funding might seem unrealistic at a time of financial stress, it should be considered that TB prevention would be many times cheaper than the current - unsuccessful - strategy of treating the disease as it is found. Consequently, failure to invest in TB control now will cost us dearly in the future. Many public-private initiatives are currently working in the right direction. The Global Laboratory Initiative is leading plans for a large expansion of laboratory services for TB, with partners such as UNITAID and PEPFAR providing tens of millions of dollars in funding for a program called EXPAND-TB that will supply rapid MDR-TB diagnostics to 27 high-burden countries [20]. A "TB Research Movement" has been conceived to accelerate progress, the goal of which is to stimulate investments, support efforts, accelerate implementation, and expand the existing portfolio across the research continuum to ensure development of those tools that will be conducive to global elimination of TB by 2050 [2]. Its objectives are to provide leadership and advocacy that will mobilize increased resources in support of a coherent and comprehensive global TB research agenda and to provide a forum for funders and implementers of TB research to coordinate plans and actions, with the result of ensuring that research needs are addressed, opportunities prioritized, and gaps filled. [2] Finally, emerging economies such as Brazil, Russia, India, and China are taking action to increase their investments in tuberculosis, realizing that (i) they account for a large proportion of the global tuberculosis burden, and (ii) locally-manufactured, low-cost tuberculosis diagnostics are viable products for their emerging pharmaceutical industries.

The "Immunodiagnosis of Tuberculosis: New Questions, New Tools" conference successfully fostered interactions between basic scientists and clinical investigators, research and implementation communities, and academia and industry. It also included participation of government agencies, TB research and development partnerships, intergovernmental organizations, and funding agencies promoting research and policy making in global health. Furthermore, the participation of a diverse audience from a global arena made it possible to convey 
within the conference viewpoints and needs expressed from worldwide communities. Similar future initiatives are expected to greatly contribute to attracting researchers in academia and industry to TB research, to fostering communication between research and implementation communities, and to promoting formation of intergovernmental working groups to tackle neglected areas that are critical for TB control.

\section{Acknowledgements}

The conference was made possible by the generous support of the following sponsors: Bill \& Melinda Gates Foundation, Foundation for Innovative New Diagnostics (FIND), the Special Programme for Research \& Training in Tropical Diseases (TDR) at the World Health Organization, and the National Institute of Allergy and Infectious Diseases.

We thank the Meeting Scientific Committee (MSC) for the meeting for helping create a novel, interdisciplinary program and contribute to the success of the conference. The MSC members were: Philip L. Felgner, University of California, Irvine, USA; Anneke Hesseling of Stellenbosch University, Stellenbosch, South Africa; Stefan H.E. Kaufmann, Max Planck Institute for Infection Biology, Berlin, Germany; Richard Menzies, McGill University, Montreal, Quebec, Canada; Mario Raviglione, Stop TB World Health Organization, Geneva, Switzerland; Mario Roederer, National Institutes of Health, Bethesda, Maryland, USA; and Douglas Young, Imperial College, London, United Kingdom.

\section{Author details}

'Public Health Research Institute, New Jersey Medical School, Newark, New Jersey, USA . ${ }^{2}$ Statens Serum Institute, Copenhagen, Denmark.

\section{Competing interests}

The authors declare that they have no competing interests.

Published: 17 December 2010

\section{References}

1. Pathways to better diagnostics for tuberculosis: A blueprint for the development of TB diagnostics. World Health Organization. Stop TB Dept. Geneva; 2009, ISBN: 9789241598811.

2. Raviglione MC: Tuberculosis is a global health issue: challenges and need for new tools. BMC Proceedings 2010

3. Young D: The diversity of latent TB. BMC Proceedings 2010

4. Wenk M: Lipidomics in biomarker development. BMC Proceedings 2010.

5. Gagnuex S: Evolutionary forces in Mycobacterium tuberculosis: implications for product development. BMC Proceedings 2010.

6. McFadden J: Systems approaches to uncovering in vivo state of the TB bacillus. BMC Proceedings 2010

7. Elledge S: A functional genomics approach to viral-host interactions for HIV. BMC Proceedings 2010.

8. Kauffman SHE: Host response to tuberculosis as basis for rational design of vaccines and biomarkers. BMC Proceedings 2010.

9. Doherty TM: Separating latent and acute TB. BMC Proceedings 2010.

10. Gennaro ML: Genome-scale antibody responses in TB. BMC Proceedings 2010.

11. Coen M, Holmes E: Translation of Metabolite Profiling to Infectious Diseases. BMC Proceedings 2010.

12. Bellanti J: The child's immune system and pediatric tuberculosis. $B M C$ Proceedings 2010.

13. Hesseling A: Pediatric tuberculosis: clinical and epidemiological reflections from a highly endemic setting. BMC Proceedings 2010.

14. Menzies R: Evaluating new diagnostic tests in the field - are we doing it right? BMC Proceedings 2010.

15. Arend S: A specific skin test: the best for both worlds? BMC Proceedings 2010.

16. Andersen P: IGRA based diagnosis of infection and prediction of disease. BMC Proceedings 2010.
17. Roederer M: Multifunctional analysis of antigen-specific $T$ cells: correlates of vaccine efficiency. BMC Proceedings 2010.

18. Lee A: Novel microfluidic technologies for portable diagnostics systems. BMC Proceedings 2010.

19. Stop TB Partnership.. TB Research Movement 2008 [http://www.stoptb. org/global/research/].

20. World Health Organization: Rapid tests for drug-resistant TB to be available in developing countries. 2008 [http://www.who.int/tb/ features_archive/mdrtb_rapid_tests/en/index.html].

doi:10.1186/1753-6561-4-S3-11

Cite this article as: Gennaro and Doherty: Immunodiagnosis of tuberculosis: new questions, new tools conference 2008. BMC Proceedings 2010 4(Suppl 3):11.

\section{Submit your next manuscript to BioMed Central and take full advantage of:}

- Convenient online submission

- Thorough peer review

- No space constraints or color figure charges

- Immediate publication on acceptance

- Inclusion in PubMed, CAS, Scopus and Google Scholar

- Research which is freely available for redistribution 